Let $O$ be the circumcentre of the triangle $A B C$, and $R$ the circumcentre of $A P Q$. Then $R O$ has equal projections on $A B$ and $\mathrm{AC}$ (each being $\frac{1}{2} \mathrm{BP}$ ); and hence $\mathrm{RO}$ is parallel to the bisector of the angle BAC. Therefore the locus of $\mathrm{R}$ is a straight line through $\mathrm{O}$, parallel to the bisector of the angle $\mathrm{BAC}$; and thus the circumcircle of $A P Q$ always passes through a fixed point $A^{\prime}$, which is the image of $A$ in $R O$. Hence, by the proposition stated above, $P Q$ envelopes a parabola of which $A^{\prime}$ is the focus, and which touches $\mathrm{AB}$ and $\mathrm{AC}$.

It is obvious from the above also that if any circle through $\mathbf{A}$ and $A^{\prime}$ meets $A B$ and $A C$ in $P$ and $Q$, then $B P=B Q$; and the following more general theorem may also be proved very easily :-

If a series of circles pass through two fixed points $A$ and $A^{\prime}$, and if two straight lines $A P Q R \ldots$ and $A P^{\prime} Q^{\prime} R^{\prime} \ldots$ meet these circles in the points $P, Q, R, \ldots$ and $P^{\prime}, Q^{\prime}, R^{\prime}, \ldots$, then $P Q: Q R: \ldots$ $=\mathbf{P}^{\prime} \mathbf{Q}^{\prime}: \mathbf{Q}^{\prime} \mathbf{R}^{\prime}$ :

This theorem may also be got by inversion; it may in fact be got by inverting the theorem that the anharmonic ratio of a pencil is constant, by taking the particular case of a number of concurrent transversals.

Third Meeting, January 9th, 1891.

R. E. Allardice, Esq., President, in the Chair.

On the Heating of Conductors by Electric Currents, and the Electric Distribution in Conductors so heated.

By Joun M'Cowan, M.A., B.Sc.

The solution of the equation.

$$
\sqrt{ }\{(x-a)(x-b)\}+\sqrt{ }\}(x-c)(x-d)\}=e \text {. }
$$

By J. D. Hamilton Dickson, M.A.

1. If $\mathbf{P}=a+b-c-d$, and $\mathbf{K}=c d-a b$, the solution of the equation

$$
\sqrt{ }\{(x-a)(x-b)\}+\sqrt{ }\{(x-c)(x-d)\}=e \quad \ldots
$$

may be most readily found by putting $(x-a)(x-b)=z^{2}$; whence 
$(x-c)(x-d)=z^{2}+\mathrm{P} x+\mathrm{K}$, and therefore $=z^{2}-2 e z+e^{2}$, and finally $x$ is given by the equation

$\left(\mathbf{P}^{2}-4 e^{2}\right) x^{2}+2\left\{\mathbf{P}\left(\mathbf{K}-e^{2}\right)+2 e^{2}(a+b)\right\} x+\left(\mathbf{K}-e^{2}\right)^{2}-4 e^{2} a b=0$

In order to simplify the discussion of this result $I$ use the following notation :-

$$
\begin{array}{lll}
\mathbf{P}=a+b-c-d, & a=(a-c)(a-d), & \kappa=(a-b)(c-d), \\
\mathbf{Q}=a-b+c-d, & \beta=(b-c)(b-d), & \lambda=(a-c)(b-d), \\
\mathbf{R}=a-b-c+d, & \gamma=(c-a)(c-b), & \mu=(a-d)(b-c) ; \\
& \delta=(d-a)(d-b), & \\
\Pi=\mathbf{P}^{2}-4 e^{2}, & \mathbf{L}=e^{2}-\lambda, & \mathbf{M}=e^{2}-\mu .
\end{array}
$$

The solution of (2) may now be written in the form

$$
\begin{gathered}
\Pi x=-\mathbf{P}\left(\mathrm{K}-e^{2}\right)-2 e^{2}(a+b) \\
\pm \sqrt{ }\left[\left\{\mathbf{P}\left(\mathbf{K}-e^{2}\right)+2 e^{2}(a+b)\right\}^{2}-\Pi\left\{\left(\mathbf{K}-e^{2}\right)^{2}-4 e^{2} a b\right\}\right],
\end{gathered}
$$

which, after some simplifications, becomes

$$
\Pi x=-e^{2}\{-\mathrm{P}+2(a+b)\}-\mathrm{PK} \pm 2 e \sqrt{ }(\mathrm{LM})
$$

whence

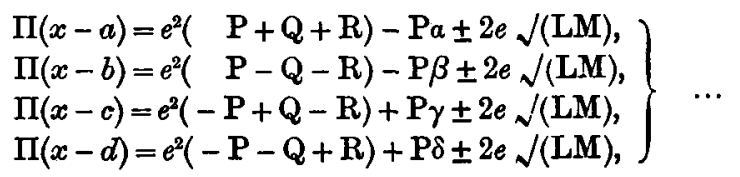

2. It is worth while verifying that the results in (4) satisfy (1). Multiplying together the first two,

$$
\begin{aligned}
\Pi^{2}(x-a)(x-b) & =e^{4}\{\mathrm{P}+2(a-b)\}\{\mathrm{P}-2(a-b)\} \\
& -e^{2} \mathrm{P}[\{\mathrm{P}+2(a-b)\} \beta+\{\mathrm{P}-2(a-b)\} a] \\
& +\mathrm{P}^{2} a \beta+4 e^{2} \mathrm{LM} \pm 2 e\left\{2 \mathrm{P} e^{2}-\mathrm{P}(a+\beta)\right\} \sqrt{ }(\mathrm{LM}) \\
& =4 e^{6}+e^{4}\left\{\mathrm{P}^{2}-4(a-b)^{2}-4 \lambda-4 \mu\right\} \\
& +e^{2}\left\{-\mathrm{P}^{2}(a+\beta)+2 \mathrm{P}(a-b)(a-\beta)+4 \lambda \mu\right\} \\
& +\mathrm{P}^{2} a \beta \pm 2\left\{2 e^{8}-(a+\beta) e\right\} \mathrm{P} \sqrt{ }(\mathrm{LM})
\end{aligned}
$$

or, noting that $\lambda \mu=\alpha \beta$, and that $\alpha-\beta=\mathrm{P}(a-b)$, and making some further reductions,

$$
\begin{aligned}
\Pi^{2}(x-a)(x-b) & =4 e^{6}-4(\alpha+\beta) e^{4}+(\alpha+\beta)^{2} e^{2} \\
+ & \mathrm{P}^{2} e^{4}-\mathrm{P}^{2}(\lambda+\mu) e^{2}+\mathrm{P}^{2} \lambda \mu \pm 2\left\{2 e^{3}-(a+\beta) e\right\} \mathrm{P} /(\mathrm{LM}),
\end{aligned}
$$

and therefore,

$$
\pm \Pi \sqrt{ }\{(x-a)(x-b)\}=2 e^{3}-(a+\beta) e \pm \mathrm{P} \sqrt{ }(\mathrm{LM}) . \quad \ldots
$$

Similarly,

$$
\pm \Pi \sqrt{ }\{(x-c)(x-d)\}=2 e^{3}-(\gamma+\delta) e \mp \mathrm{P} /(\mathbf{L M}) ; \quad \ldots
$$

whence, 


$$
\begin{aligned}
\Pi[\sqrt{ }\{(x-a)(x-b)\}+\mathcal{V}\{(x-c)(x-d)\}] & =4 e^{3}-(\alpha+\beta+\gamma+\delta) e, \\
& = \pm \Pi e,
\end{aligned}
$$

since $a+\beta+\gamma+\delta=\mathrm{P}^{2}$.

3. This verification suggests the possibility of $\Pi(x-a)$ being also expressible in the form of a square. In the case of $\mathrm{A}+\sqrt{ } \mathbf{C}$ being the same as $(\sqrt{ } u+\sqrt{ } v)^{2}$, and $\mathrm{B}+\sqrt{\mathrm{C}}$ being the same as $\left(\sqrt{ } u^{\prime}+\sqrt{ } v^{\prime}\right)^{2}$, it follows at once that $(A+\sqrt{ } \mathbf{C})(B+\sqrt{ } \mathbf{C})$ is of a similar form. But the converse is not true. The necessary condition is that $\left(A^{2}-C\right)$ $\left(B^{2}-C\right)$ be a complete square, which is satisfied if

$$
\mathrm{A}^{2}-\mathrm{C}=\mathrm{F}^{2} \mathrm{\theta} \text {, and } \mathrm{B}^{2}-\mathrm{C}=\mathrm{G}^{2} \Theta \text {. }
$$

To examine the case for $\pi(x-a)$, I look for the factors of

$$
\left\{e^{2}(\mathrm{P}+\mathrm{Q}+\mathrm{R})-\mathrm{P} \alpha\right\}^{2}-4 e^{2} \mathrm{LM} \text {. }
$$

Changing the sign of this expression, for a moment, it

$$
\begin{aligned}
& =4 e^{2}\left(e^{2}-\lambda\right)\left(e^{2}-\mu\right)-\left[\{\mathbf{P}+2(a-b)\} e^{2}-\mathbf{P} a\right]^{2} \\
& =4 e^{6}-e^{4}\left\{\mathrm{P}^{2}+4 \mathrm{P}(a-b)+4(a-b)^{2}+4 \lambda+4 \mu\right\} \\
& +e^{2}\left\{2 \mathrm{P}^{2} a+4 \mathrm{P} \alpha(a-b)+4 \lambda \mu\right\}-\mathrm{P}^{2} a^{2} \\
& =4 e^{8}-e^{4}\left(\mathrm{P}^{2}+8 a\right)+e^{2}\left(2 \mathrm{P}^{2} \alpha+4 a^{2}\right)-\mathrm{P}^{2} \alpha^{2} \\
& =\left(4 e^{2}-\mathrm{P}^{2}\right)\left(e^{2}-a\right)^{2} \\
& =-\Pi\left(e^{2}-a\right)^{2} \text {. }
\end{aligned}
$$

Hence

$$
\begin{aligned}
\Pi(x-a)= & e^{2}(\mathrm{P}+\mathrm{Q}+\mathrm{R})-\mathrm{P} a \pm 2 e \sqrt{ }(\mathrm{LM}) \\
= & {\left[\sqrt{ }\left\{\frac{1}{2}(\mathrm{P}+\mathrm{Q}+\mathrm{R}) e^{2}+\frac{1}{2}\left(e^{2}-a\right) \sqrt{ } \Pi\right\}\right.} \\
& \left.+\sqrt{ }\left\{\frac{1}{2}(\mathrm{P}+\mathrm{Q}+\mathrm{R}) e^{2}-\frac{1}{2}\left(e^{2}-a\right) \sqrt{ } \Pi\right\}\right]^{2},
\end{aligned}
$$

and similarly for the other expressions in (4).

The usefulness of such a form as (8) depends on its freedom from radicals Two of such cases may be noted. Let $e^{2}=\lambda$, then

$$
\Pi=\mathbf{P}^{2}-4 \lambda=(a-b-c+d)^{2}=\mathbf{R}^{2}
$$

and

$$
\lambda-a=-(a-b)(a-c),
$$

whence

$$
\begin{aligned}
& x-a=-(a-b)(a-c) / \mathbf{R}, \\
& x-b=-(a-b)(b-d) / \mathbf{R}, \\
& x-c=-(a-c)(c-d) / \mathbf{R}, \\
& x-d=-(b-d)(c-d) / \mathbf{R} .
\end{aligned}
$$

Similarly, when $e^{2}=\mu$, we have $\Pi=Q^{2}$, and

$$
\begin{aligned}
& x-a=-(a-b)(a-d) / \mathrm{Q}, \\
& x-b=-(a-b)(b-c) / \mathrm{Q}, \\
& x-c=-(b-c)(c-d) / \mathrm{Q}, \\
& x-d=(a-d)(c-d) / \mathrm{Q} .
\end{aligned}
$$


These are what we get from the vanishing of $L$ and $M$ in the general solution. Other four cases are presented in this form of the solution, viz., when $e^{2}=\alpha, \beta, \gamma, \delta$; but these will be referred to further on, under a new aspect.

4. Thus treated, the complete solution of the general equation is given in terms of $e$. A case of more than usual interest arises when $\Pi=0$, or $\mathrm{P}^{2}=4 e^{2}$. The equation then takes one of two forms, either

$$
\begin{aligned}
& 2 \sqrt{ }\{(x-a)(x-b)\}+2 \sqrt{ }\{(x-c)(x-d)\}=a+b-c-d \\
& =-(x-a)-(x-b)+(x-c)+(x-d) \text {, } \\
& \text { whence } \\
& \sqrt{ }(x-a)+\sqrt{ }(x-b)+\sqrt{ }(x-c)-\sqrt{ }(x-d)=0,\} \\
& \sqrt{ }(x-a)+\sqrt{ }(x-b)-\sqrt{ }(x-c)+\sqrt{ }(x-d)=0 ;\} \\
& \text { or (II.) } 2 \sqrt{ }\{(x-a)(x-b)\}+2 \sqrt{ }\{(x-c)(x-d)\}=-a-b+c+d \text {, } \\
& \text { whence } \quad \sqrt{(x-a)-\sqrt{ }(x-b)+\sqrt{ }(x-c)+\sqrt{ }(x-d)=0,}\} \\
& \text { or } \quad-\sqrt{ }(x-a)+\sqrt{ }(x-b)+\sqrt{ }(x-c)+\sqrt{ }(x-d)=0 .\}
\end{aligned}
$$$$
\text { or }
$$

The solution is got from (2) at once, in the form

or,

$$
x=\frac{4 e^{2} a b-\left(\mathrm{K}-e^{2}\right)^{2}}{4 e^{2}(a+b)+2 \mathbf{P}\left(\mathbf{K}-e^{2}\right)},
$$

whence

$$
\begin{array}{lll}
x=\left\{\mathbf{P}^{2} a b-\left(\mathrm{K}-\frac{1}{2} \mathrm{P}^{2}\right)\right\} / \frac{1}{2} \mathrm{PQR} & \ldots & \ldots \\
x-a=-\left(e^{2}-a\right)^{2} / \frac{1}{2} \mathrm{PQR}, & & \\
x-b=-\left(e^{2}-\beta\right)^{2} / \frac{1}{2} \mathrm{PQR}, & & \\
x-c=-\left(e^{2}-\gamma\right)^{2} / \frac{1}{2} \mathrm{PQR}, & \ldots & \ldots \\
x-d=-\left(e^{2}-\delta\right)^{2} / \frac{1}{2} \mathrm{PRR} . & &
\end{array}
$$

These results agree with equations (9); for, irrespective of sign, sum of square roots of numerators $=4 e^{2}-(\alpha+\beta+\gamma+\delta)=0$.

The results in (11) are remarkable as containing the complete and (as will appear afterwards) the unique solution of the following problem in Diophantine Analysis :-

To find integral values for $\mathrm{p}$ and $\mathrm{q}$ to satisfy the conditions that ap $-\mathrm{q}, \mathrm{bp}-\mathrm{q}, \mathrm{cp}-\mathrm{q}, \mathrm{dp}-\mathrm{q}$ are all positive squares.

The solution holds if any one, two, or three of the quantities $a, b, c, d$ is zero ; they may be positive or negative : but obviously the solution fails if $P, Q$, or $R$ vanish.

5. Further insight into the properties of the equation considered may be obtained by geometry.

Let $\mathrm{O}, \mathrm{A}, \mathrm{B}, \mathrm{C}, \mathrm{D}$ (fig. 8), be five points (in order) on a straight line, and if $a, b, c, d$ be in ascending order of magnitude they may 
be taken as the numerical values of the length $O A, O B, O C, O D$. If $x$ be the corresponding alsscissa of some point $\mathrm{X}$ on the line $\mathrm{OD}$, then, for $e$ to be real, obviously $\mathrm{X}$ must lie outside of both the circles described on $\mathrm{AB}$ and $\mathrm{CD}$ as diameters. Let $\mathrm{G}, \mathrm{H}$ be the centres, respectively, of these circles; and let the common tangents $\mathrm{EF}, \mathrm{E}^{\prime} \mathrm{F}^{\prime}$ cut the line $\mathrm{GH}$ (produced if necessary) in $\mathrm{X}_{1}, \mathrm{X}_{1}^{\prime}$ respectively : then $X_{1}, X_{1}^{\prime}$ are the centres of similitude of these circles. Hence we find,

$$
\left.\begin{array}{rlrl}
\mathbf{G H}=\mathrm{OH}-\mathrm{OG} & =-\frac{1}{2} \mathrm{P}, & \mathrm{OX}_{1} & =(a d-b c) / \mathrm{R}, \\
\mathbf{F H}-\mathbf{E G}=\frac{1}{2} \mathbf{R}, & \mathrm{OX}_{1}^{\prime}=(a c-b d) / \mathrm{Q} . \\
\mathbf{F}^{v} \mathrm{H}+\mathbf{E}^{\prime} \mathrm{G}=-\frac{1}{2} \mathrm{Q}, & \mathrm{X}_{1} \mathrm{X}_{1}^{\prime}=\mathrm{P}_{\kappa} / \mathrm{QR} .
\end{array}\right\} \quad \ldots
$$

Now, let us suppose $x=\mathrm{OX}_{1}$, then

$$
\begin{aligned}
& \left.\begin{array}{l}
\mathbf{X}_{\mathbf{1}} \mathbf{E}=\sqrt{ }\{(x-a)(x-b)\}=(a-b) \sqrt{ } \lambda / \mathbf{R} \\
\mathbf{X}_{1} \mathbf{F}=\sqrt{ }\{(x-c)(x-d)\}=(c-d) \sqrt{ } \lambda / \mathbf{R}
\end{array}\right\} \quad \ldots \\
& e=\mathrm{X}_{1} \mathrm{E}+\mathrm{X}_{1} \mathrm{~F}=\mathrm{Q} \sqrt{ } / \lambda / \mathrm{R} \\
& \text {... }
\end{aligned}
$$

Again, if $x=\mathrm{OX}_{1}^{\prime}$, then

whence

$$
\mathrm{X}_{1}^{\prime} \mathbf{E}^{\prime}=(a-b) \sqrt{ } \mu / \mathrm{Q}, \quad \mathrm{X}_{1}^{\prime} \mathrm{F}^{\prime}=(c-d) \sqrt{ } \mu / \mathrm{Q}, \quad \ldots
$$

whence

$$
\left.\begin{array}{rl}
e=\mathrm{X}_{1}^{\prime} \mathrm{E}^{\prime}+\mathrm{X}_{1}^{\prime} \mathrm{F}^{\prime} & =\sqrt{ } \mu \\
e=-\mathrm{X}_{1}^{\prime} \mathrm{E}^{\prime}+\mathrm{X}_{1}^{\prime} \mathrm{F}^{\prime} & =-\mathrm{R} \sqrt{ } \mu / \mathrm{Q}
\end{array}\right\} \quad \cdots
$$

6. Let, next, the numerical quantities $a, b, c, d$ be arranged in a new order, under the signs of the square root-viz., the first and third under one root, and the second and fourth under the other root, so that the equation is now

$$
\sqrt{ }\{(x-a)(x-c)\}+\sqrt{ }\{(x-b)(x-d)\}=e .
$$

For a real value of $e, \mathbf{X}$ must lie outside of the circles (Fig. 9) described on $\mathrm{AC}$, and $\mathrm{BD}$ as diameters. Here, again, we find the values,

$$
\left.\begin{array}{rl}
\mathrm{GH}=\mathrm{OH}-\mathrm{OG}=-\frac{1}{2} \mathrm{Q}, \quad \mathrm{OX}_{2}=(a d-b c) / \mathrm{R} \\
\mathrm{FH}-\mathrm{EG}=\frac{1}{2} \mathrm{R}, \quad \mathrm{OX}_{2}^{\prime}=(a b-c d) / \mathrm{P}, \\
\mathbf{F H}+\mathrm{EG}=-\frac{1}{2} \mathrm{P}, \quad \mathrm{X}_{2} \mathrm{X}_{2}^{\prime}=\mathrm{Q} \lambda / \mathrm{PR} ;
\end{array}\right\}
$$

and if $x=\mathrm{OX}_{2}$, then

$$
\begin{aligned}
& \left.\begin{array}{l}
\mathrm{X}_{2} \mathrm{E}=\sqrt{ }\{(x-a)(x-c)\}=(a-c) \sqrt{ } \kappa / \mathrm{R} \\
\mathrm{X}_{2} \mathrm{~F}=\sqrt{ }\{(x-b)(x-d)\}=(b-d) \sqrt{ } \kappa / \mathrm{R}
\end{array}\right\} \quad \ldots \\
& \left.\begin{array}{l}
e=\mathrm{X}_{2} \mathrm{E}+\mathrm{X}_{2} \mathrm{~F}=\mathrm{P} \sqrt{ } \kappa / \mathrm{R}, \\
e=-\mathrm{X}_{2} \mathrm{E}+\mathrm{X}_{2} \mathrm{~F}=-\sqrt{ } \kappa
\end{array}\right\} \quad \ldots
\end{aligned}
$$


Also, if $x=\mathrm{OX}_{2}^{\prime}$, then

$$
\mathrm{X}_{2}^{\prime} \mathrm{E}^{\prime}=i(a-c) \sqrt{ } \mu / \mathrm{P}, \quad \mathrm{X}_{2}{ }^{\prime} \mathrm{F}^{\prime}=i(b-d) \sqrt{ } \mu / \mathrm{P} ; \quad \ldots
$$

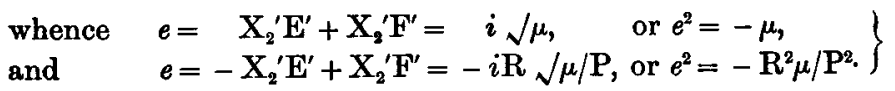

7. Finally, arranging the quantities $a, b, c, d$, so that the equation takes the form $\sqrt{ }\{(x-a)(x-d)\}+\sqrt{ }\{(x-b)(x-c)\}=e$; in order that $e$ may be real, $\mathrm{X}$ must lie outside of the circle (fig. 10), described on $\mathrm{AD}$ as diameter. As before we find

$$
\left.\begin{array}{rlrl}
\mathbf{G H}=\mathrm{OH}-\mathrm{OG}=-\frac{1}{2} \mathrm{R}, & \mathrm{OX}_{3}=(a c-b d) / \mathrm{Q}, \\
& \mathbf{F H}-\mathbf{E G}=\frac{1}{2} \mathrm{Q}, & \mathrm{OX}_{3}^{\prime}=(a b-c d) / \mathrm{P}, \\
\mathbf{F H}+\mathbf{E G}=-\frac{1}{2} \mathbf{P} ; & \mathrm{X}_{3} \mathrm{X}_{3}^{\prime}=\mathbf{R} \mu / \mathbf{P Q} .
\end{array}\right\}
$$

If $x=\mathrm{OX}_{3}$; then

$$
\left.\begin{array}{l}
\mathrm{X}_{3} \mathrm{E}=\sqrt{ }\{(x-a)(x-d)\}=i(a-d) \sqrt{ } \kappa / \mathrm{Q} \\
\mathrm{X}_{3} \mathrm{~F}==\sqrt{ }\{(x-b)(x-c)\}=i(b-c) \sqrt{ } \kappa / \mathrm{Q}
\end{array}\right\}
$$

$\left.\begin{array}{ll}\text { whence, } & e=\mathrm{X}_{3} \mathrm{E}+\mathrm{X}_{3} \mathrm{~F}=i \mathrm{P} \sqrt{ } \kappa / \mathrm{Q} ; \text { or, } e^{2}=-\mathrm{P}^{2} \kappa / \mathrm{Q}^{2} ; \\ \text { and } & e=-\mathrm{X}_{3} \mathrm{E}+\mathrm{X}_{3} \mathrm{~F}=-i \sqrt{ } \kappa ; \quad \text { or, } e^{2}=-\kappa .\end{array}\right\}$

Also, if $x=\mathrm{OX}_{3}^{\prime}$, we get

$$
\mathrm{X}_{3} \mathbf{E}^{\prime}=i(a-d) \sqrt{ } \lambda / \mathrm{P} ; \mathrm{X}_{3}^{\prime} \mathbf{F}^{\prime}=i(b-c) \sqrt{ } \lambda / \mathbf{P} \quad \ldots
$$

whence,

$$
\left.\begin{array}{l}
e=\mathrm{X}_{\mathbf{3}}{ }^{\prime} \mathrm{E}^{\prime}+\mathrm{X}_{3}{ }^{\prime} \mathrm{F}^{\prime}=i \sqrt{ } \lambda ; \quad \text { or, } e^{2}=-\lambda: \\
e=-\mathrm{X}_{3}^{\prime} \mathrm{E}^{\prime}+\mathrm{X}_{3}^{\prime} \mathrm{F}^{\prime}=-i \mathrm{Q} \sqrt{ } \lambda / \mathbf{P} ; \text { or, } e^{2}=-Q^{2} \lambda / \mathrm{P}^{2} .
\end{array}\right\}
$$

The above results lead to several geometrical theorems, e.g.:-

If $A, B, C, D$ be four points in order on a straight line, and if pairs of circles be described on $\mathrm{AB}, \mathrm{CD}$ as diameters ; on $\mathrm{AC}, \mathrm{BD}$ as diameters; and on $\mathrm{AD}, \mathrm{BC}$ as diameters : the three corresponding pairs of centres of similitude will be represented by only three points.

The length of the common external tangent to the circles on $A B$

- The results of $\$ \$ 6,7$, might have been derived by substitution from those of $\S 5$. Thus we get $\S 6$ by the substitution $\begin{aligned} & b, c \\ & c, b\end{aligned}$, which gives

and $\S 7$ by the substitution

$$
\begin{aligned}
& \mathbf{P}, \mathbf{Q}, \mathbf{R} \text { and } \kappa, \lambda,{ }^{\mu} \\
& \mathbf{Q}, \mathbf{P}, \mathbf{R} \quad \lambda_{,} \kappa_{1}-\mu \\
& b, c, d, \text { which gives } \\
& d, b c, \text {, w }
\end{aligned}
$$

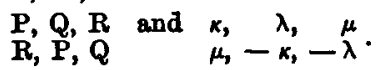


and $C D$ is equal to one of the intercepts on the chord, perpendicular to $\mathrm{AD}$, made by the circles on $\mathrm{AD}$ and $\mathrm{BC}$.

8. The properties of the equation may also be studied with advantage in the form

$$
\sqrt{ }\{(x-a)(x-b)\}+\sqrt{ }\{(x-c)(x-d)\}=y .
$$

Figs 11, 12, 13 represent the equations

and

$$
\begin{aligned}
& \mp \sqrt{ }\{(x-1)(x-2)\}+\sqrt{ }\{(x-5)(x-7)\}=y \\
& \mp \sqrt{ }\{(x-1)(x-5)\}+\sqrt{ }\{(x-2)(x-7)\}=y \\
& \mp \sqrt{ }\{(x-1)(x-7\})+\sqrt{ }\{(x-2)(x-5)\}=y
\end{aligned}
$$

the upper sign referring to the full lines, the lower to the totted lines.

For the upper signs, $\pm y=\frac{1}{2} \mathrm{P}$ are the asymptotes ; and, for the lower signs, $\pm y=2\left(x-\frac{a+b+c+d}{4}\right)$ are the symptotes : viz,

in fig. 11, in fig. 12, in fig. 13 ,

$$
\begin{aligned}
& \pm y=4 \frac{1}{2}, \text { and } \pm y=2\left(x-3 \frac{3}{4}\right) \\
& \pm y=1 \frac{1}{2}, \text { and } \pm y=2\left(x-3 \frac{3}{4}\right) \\
& \pm y=\frac{1}{2}, \text { and } \pm y=2\left(x-3 \frac{3}{4}\right)
\end{aligned}
$$

In fig. 11 the curve consists of three separate portions lying in the regions (1) from $x=-\infty$ to $x=1$; (2) from $x=2$ to $x=5$; and (3) from $x=7$ to $x=\infty$. In the first branch there is a point of inflexion at $x=-6 \cdot 2 \ldots$, and in the second there is another point of inflexion at $x=3.2 \ldots$, both points being given by the equation

$$
(1-m) x^{2}-\{(a+b)-m(c+d)\} x+a b-m c d=0
$$

where $m=(a-b)^{3} /(c-d)^{3}$. There is a minimum value of $y$ in the full curve, at $x=-3$ (viz., $y=\sqrt{ } \lambda=\sqrt{ } 20$ ); and a maximum value of $y$, in the dotted curve, at $x=3$ (viz., $y=\sqrt{ } \mu=\sqrt{ } 18$ ).

In fig. 12 the middle portions of both curves have disappeared (become imaginary). There is a point of inflexion at $x=-5 \cdot 47 \ldots$; a minimum value of $y$ at $x=-3$ (viz., $y=\sqrt{ } \kappa=\sqrt{ } 2$ ). The other point of inflexion and the maximum point have disappeared.

In fig. 13 the full curve, in its right branch, changes from the negative to the positive region of $y$; and the maximum and minimum points have disappeared as well as the points of inflexion. 
9. Thus I prove that, if $a, b, c, d$ are in ascending order of magnitude (algebraical), the equation

$$
\mp \sqrt{ }\{(x-a)(x-b)\}+\sqrt{ }\{(x-c)(x-d)\}=e
$$

has two real roots in the following cases,

(1) for the upper sign, if $e$ lie between $\sqrt{ } \lambda$ and $-\frac{1}{2} \mathrm{P}$;

(2) for the lower sign, if $e$ lie between $\sqrt{ } \beta$ and $\sqrt{\mu}$,or $\Varangle \sqrt{ } \delta$;

(3) one for each sign, if $e$ lie between $\sqrt{ } \lambda$ and $\sqrt{ } \beta$ : that it has one real root, and only one, in the following cases,

(1) for the upper sign, if $e$ lie between $-\sqrt{ } \delta$ and $\frac{1}{2} \mathrm{P}$,

$$
\begin{array}{r}
\text { between }-\sqrt{ } \gamma \text { and }+\sqrt{ } \gamma, \\
\text { or between }-\frac{1}{2} P \text { and } \sqrt{ } \alpha
\end{array}
$$

(2) for the lower sign, if $e$ lie between $\sqrt{ } a$ and $\sqrt{ } \delta$ : and, finally, that the roots are imnginary,

$$
\begin{aligned}
& \text { if } e \text { be }<-\sqrt{\delta}, \\
& \text { or if } e \text { lie between } \frac{1}{2} P \text { and }-\sqrt{\gamma}, \\
& \text { or between } \sqrt{ } \mu \text { and } \sqrt{\lambda} .
\end{aligned}
$$

The critical cases, when $e^{2}=\lambda, \mu, \alpha, \beta, \gamma$, or $\delta$ are too simple to require consideration.

The equations

and

$$
\begin{aligned}
& \mp \sqrt{ }\{(x-a)(x-c)\}+\sqrt{ }\{(x-b)(x-d)\}=e, \\
& \mp \sqrt{ }\{(x-a)(x-d)\}+\sqrt{ }\{(x-b)(x-c)\}+e,
\end{aligned}
$$

may be discussed in a similar manner, and lead to similar, but simpler, results.

10. On examining the circumstances of the numerical example considered, $\S 8$, it is found to belong to the class which, assuming $a, b, c, d$ to be in ascending order of magnitude, may be characterised by the inequalities .

$$
\delta>a>\beta>\gamma
$$

a class which is completely determined by the condition that $\mathrm{R}$ is positive. When $R$ is negative, the inequalities are

$$
a>\delta>\gamma>\beta,
$$

which is the only other class that arises. By changing the sign of $x$, and correspondingly re-srranging the values to be assigned to $a, b, c, d$, this class is reduced to the former class.

Note also that $\lambda$ is always greater than $\mu$. 
11. The circumstances of the roots of each equation may be suitably exhibited in the following tables :-

Fig. 11.

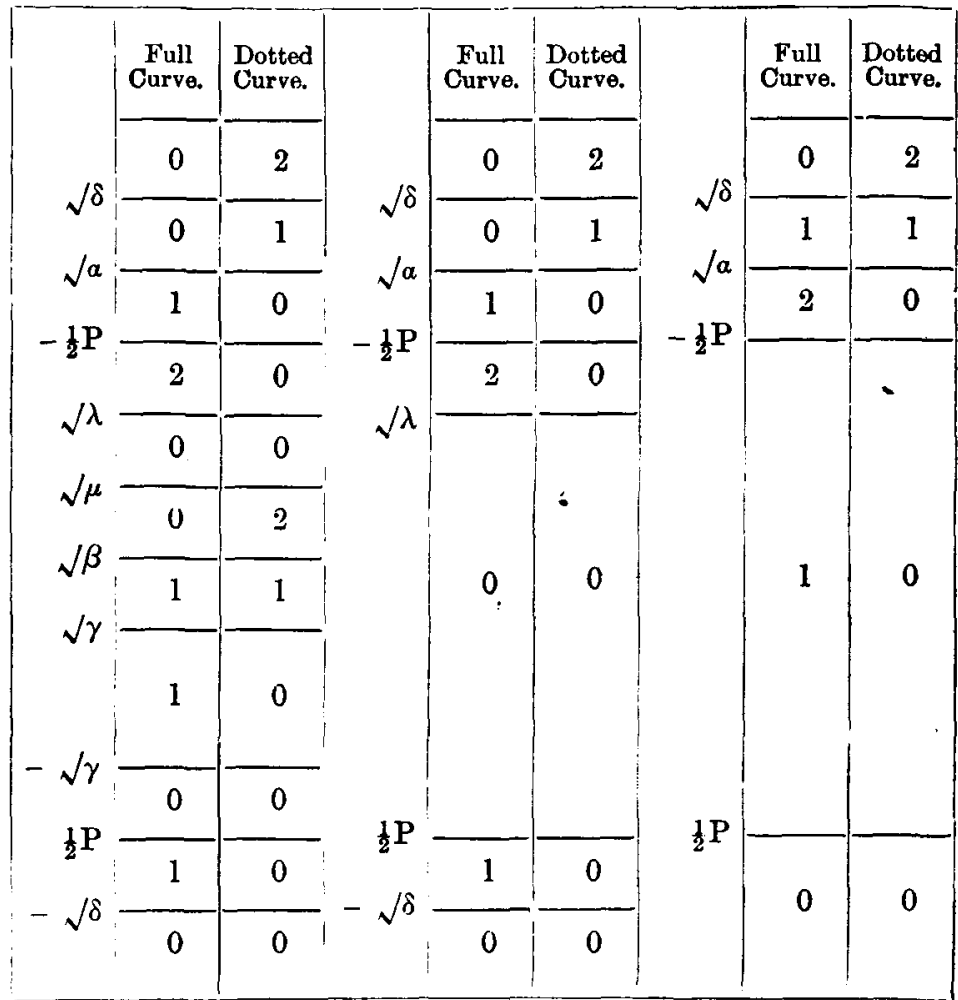

For example, in the case of an equation of the second type, if $e$ lies between $-\sqrt{ } \delta$ and $\frac{1}{2} \mathrm{P}$, there is one root for the full curve whose equation is

$$
-\sqrt{ }\{(x-a)(x-c)\}+\sqrt{ }\{(x-b)(x-d)\}=e
$$

and no root for the dotted curve, whose equation is

$$
\sqrt{ }\{(x-a)(x-c)\}+\sqrt{ }\{(x-b)(x-d)\}=e,
$$

$a, b, c, d$ being in ascending order of magnitude.

12. In solving equations of this kind it will be convenient to employ the following nomenclature:- the numbers $a, b, c, d$ may be 
called the abscissa of the equation, $a$ being, in particular, the origin : $a, \beta, \gamma, \delta$ (in preference to their square roots) may be called the terminals of the equation. To aid in simplifying the work, if in the given equation $R$ is negative, it will be advisable to change the sign of $x$ before appointing the values of the abscissæ. It will also shorten the work slightly to arrange that $a=0$, in which case $b, c, d$ will be positive.

Example.-In the following equation, written down at random,

$$
\sqrt{ }\{(x+5)(x-2)\}-\sqrt{ }\{(x-1)(x-4)\}=6,
$$

$\mathbf{R}=-4$. In order to make $R$ positive and have the origin zero, writing $-x+4$ for $x$, the equation reduces to the second typical form, viz.,

$$
-\sqrt{ }\{x(x-3)\}+\sqrt{ }\{(x-2)(x-9)\}=6,
$$

in which we have the abscissæ,

$$
\begin{aligned}
& a=0 \text { ) whence } \mathrm{P}=-8, \quad \alpha=18, \lambda=12, \quad \mathrm{~L}=24 \text {, } \\
& b=3 \quad \mathrm{Q}=-10, \quad \beta=-6, \quad \mu=-9, \quad \mathrm{M}=45 \text {, } \\
& \left.\begin{array}{lll}
c=2 \\
d=9
\end{array}\right\} \quad \mathrm{R}=4, \quad \begin{array}{ll}
\gamma=-2, & \text { II }=-80 ; \\
\delta=54, &
\end{array}
\end{aligned}
$$

and the asymptotes

$$
y= \pm 4, \text { and } y= \pm(2 x-7)
$$

As $e$ is greater than $\sqrt{ } \alpha[$ viz., $6>\sqrt{ } 18]$, there are no real roots of the equation. But, as $e$ is also less than $\sqrt{ } \delta$, the equation

$$
\sqrt{ }\{x(x-3)\}+\sqrt{ }\{(x-2)(x-9)\}=6
$$

has one real root, viz., by (4)

$$
\begin{aligned}
x & =4 \frac{1}{2} \pm(9 \sqrt{ } 30) / 10=4.5 \pm 4.9295 \ldots \\
& =9.4295 \ldots \text { or }-.4295 \ldots
\end{aligned}
$$

whence, a reference to the figure (fig. 12) shows that $-4295 \ldots$ is the required root, $9 \cdot 4295$... being the real root of the equation

$$
-\sqrt{ }\{x(x-3)\}+\sqrt{ }\{(x-2)(x-9)\}=-6,
$$

13. The following is worthy of note; taking the two equations

and

$$
-\sqrt{ }\{(x-a)(x-b)\}+\sqrt{ }\{(x-c)(x-d)\}=e
$$

then

$$
\sqrt{ }\{(x-a)(x-b)\}+\sqrt{ }\{(x-c)(x-d)\}=f
$$

or

$$
\begin{gathered}
-(x-a)(x-b)+(x-c)(x-d)=e f \\
\mathbf{P} x+\mathrm{K}=e \dot{f} .
\end{gathered}
$$


The same value of $x$ being a root of both equations we have the following theorem :-A root of the equation

$$
-\sqrt{ }\{(x-a)(x-b)\}+\sqrt{ }\{(x-c)(x-d)\}=e
$$

is also $a$ root of the equation

$$
\sqrt{ }\{(x-a)(x-b)\}+\sqrt{ }\{(x-c)(x-d)\}=\{(a+b-c-d) x-a b+c d\} / e
$$

or, elimitating $x$ by means of (4) from the right-hand side of this last equation, it is a root of the equation

$$
\begin{aligned}
& \sqrt{ }\{(x-a)(x-b)\}+\sqrt{ }\{(x-c)(x-d)\} \\
& =e\{\mathrm{P}(\mathrm{P}+\mathrm{Q}+\mathrm{R})-4 a\} / \Pi \pm 2 \mathrm{P} \sqrt{ }(\mathbf{L M}) / \Pi,
\end{aligned}
$$

the ambiguity of sign being properly chosen.

Example.-We have seen that $x=9 \cdot 4295 \ldots$ is the root of the equation

$$
-\sqrt{ }\{x(x-3)\}+\sqrt{ }\{(x-2)(x-9)\}=-6 ;
$$

whence we find that the same value of $x$ is the root of the equations

and

$$
\begin{aligned}
& \sqrt{ }\{x(x-3)\}+\sqrt{ }\{(x-2)(x-9)\}=\frac{4}{3} x-3, \\
& \sqrt{ }\{x(x-3)\}+\sqrt{ }\{(x-2)(x-9)\}=\frac{3}{5}(5+2 \sqrt{ } 30) .
\end{aligned}
$$

Fourth Meeting, February 13, 1891.

R. E. Allardice, Esq., M.A., F.R.S.E., President, in the Chair.

\section{A problem in the theory of numbers.}

By T. Hugh Miller, M.A.

Let it be required to find the square integral numbers which added to a given integer shall produce a square integer, and the smallest such number.

Let $\mathrm{N}$ be the given number, and let the sum of $\mathrm{N}$ and $x^{2}$ be $y^{2}$, where $i$ and $y$ are integers.

Then

$$
\mathrm{N}=y^{2}-x^{2}=(y+x)(y-x)
$$

Now resolve $\mathrm{N}$ into its prime factors, let these $l^{a} m^{b} n^{c} \ldots$. Then if $y+x$ is put equal to the product of any number of these factors, and $y-x$ equal to the product of all the others, a series of values of $y$ is obtained. 